\title{
CEREUS
}

DOI:10.18605/2175-7275/cereus.v9n1p.163-177

\section{ASSOCIAÇÃO ENTRE ÍNDICE DE MASSA CORPORAL E CIRCUNFERÊNCIA DA CINTURA COM HIPERTENSÃO ARTERIAL SISTÊMICA EM CAMINHONEIROS}

\author{
NOTTO, Vinícius Oliveira ${ }^{1}$ \\ BRANDÃO, Valéria de Lemos ${ }^{1,2}$ \\ ALVES, Amanda Freitas ${ }^{1}$ \\ SILVA, Loianny Moraes ${ }^{1}$ \\ D'ALESSANDRO, Walmirton Bezerra ${ }^{3}$
}

\section{RESUMO}

Objetivo: Avaliar associação entre índice de massa corporal (IMC), circunferência da cintura (CC) e a ocorrência de hipertensão arterial sistêmica (HAS) em caminhoneiros no norte do País. Método: Estudo transversal realizado com 548 caminhoneiros na BR-153. A HAS foi considerada para valores pressóricos $\geq 140 \mathrm{mmHg}$ para PA sistólica e/ou $\geq$ $90 \mathrm{mmHg}$ para PA diastólica. O IMC foi utilizado para identificar peso normal $\left(18,5-24,9 \mathrm{~kg} / \mathrm{m}^{2}\right)$, sobrepeso $(25,0$ $\left.29,9 \mathrm{~kg} / \mathrm{m}^{2}\right)$ e obesidade $\left(\geq 30 \mathrm{~kg} / \mathrm{m}^{2}\right)$. A CC foi classificada conforme o risco de complicações metabólicas. Para homens: $\geq 94 \mathrm{~cm}$ - risco aumentado e $\geq 102 \mathrm{~cm}$ - risco aumentado

\footnotetext{
${ }^{1}$ Graduandos em Medicina, Centro Universitário UnirG, Gurupi/TO.

${ }^{2}$ Nutricionista pela Pontifícia Universidade Católica de Goiás.

${ }^{3}$ Doutor em Medicina Tropical e Saúde Pública pela Universidade Federal de Goiás, professor do Centro Universitário UnirG, Gurupi/TO.
}

v.9, n. 1, jan/abr. 2017 UnirG, Gurupi, TO, Brasil 
substancialmente. Foram utilizados o teste do qui-quadrado, teste do qui-quadrado para tendência e teste exato de Fisher. A razão de chances (odds ratio) foi calculada com respectivo intervalo de confiança de 95\% utilizando regressão logística (análise realizada no Stata 12). Resultados: $81,7 \%$ dos caminhoneiros estavam com IMC $\geq 25 \mathrm{~kg} / \mathrm{m}^{2}$ e $47,8 \%$ estavam com CC $\geq 102 \mathrm{~cm}$. A HAS foi detectada em $43,6 \%$ da amostra. A regressão logística mostrou associação entre 0 excesso de peso e HAS tanto para o IMC quanto para CC. Houve aumento da probabilidade de HAS em $754 \%$ para aqueles com IMC $\geq 35 \mathrm{Kg} / \mathrm{m}^{2}$. A chance de HAS aumentou em $474 \%$ para CC $>102 \mathrm{~cm}$. Na categoria de $I M C \geq 30 \mathrm{Kg} / \mathrm{m}^{2}$, a probabilidade de HAS foi maior que aquelas observadas para os níveis da CC. Conclusão: Houve associação significativa entre os dois métodos antropométricos e a elevação da pressão arterial, indicando que quanto maior 0 excesso de peso, maior a probabilidade de HAS.

Palavras-chave: Hipertensão; Índice de Massa Corporal; Circunferência da Cintura.

\section{ASSOCIATION BETWEEN BODY MASS INDEX AND WAIST CIRCUMFERENCE WITH SYSTEMIC ARTERIAL HYPERTENSION IN TRUCK DRIVERS}

\section{ABSTRACT}

Objective: To evaluate the association between body mass index (BMI), waist circumference (WC) and the occurrence of systemic arterial hypertension (SAH) in truck drivers in the north of the country. Method: A cross-sectional study with 548 truck drivers in BR-153. SAH was considered for pressure values $\geq 140 \mathrm{mmHg}$ for systolic BP and / or $\geq 90 \mathrm{mmHg}$ for diastolic BP. BMI was used to identify normal weight (18.5$\left.24.9 \mathrm{~kg} / \mathrm{m}^{2}\right)$, overweight $\left(25.0-29.9 \mathrm{~kg} / \mathrm{m}^{2}\right)$ and obesity $(\geq 30$ 
$\mathrm{kg} / \mathrm{m}^{2}$ ). CC was classified according to the risk of metabolic complications. For males: $\geq 94 \mathrm{~cm}$ - increased risk and $\geq 102$ $\mathrm{cm}$ - risk increased substantially. The qui-square test, quisquare test for tendency and Fisher's exact test were used. The odds ratio was calculated with a respective $95 \%$ confidence interval using logistic regression (analysis performed in Stata 12). Results: $81.7 \%$ of the truck drivers had $\mathrm{BMI} \geq 25 \mathrm{~kg} / \mathrm{m}^{2}$ and $47.8 \%$ had $\mathrm{CC} \geq 102 \mathrm{~cm}$. The SAH was detected in $43.6 \%$ of the sample. Logistic regression showed an association between overweight and hypertension in both $\mathrm{BMI}$ and WC. There was an increase in the probability of $\mathrm{SAH}$ in $754 \%$ for those with $\mathrm{BMI} \geq 35 \mathrm{~kg} / \mathrm{m}^{2}$. The odds of HAS increased by $474 \%$ for $\mathrm{CC}>102 \mathrm{~cm}$. In the BMI category $\geq 30$ $\mathrm{kg} / \mathrm{m}^{2}$, the probability of SAH was higher than those observed for the CC levels. Conclusion: There was a significant association between the two anthropometric methods and elevated blood pressure, indicating that the greater the excess weight, the greater the probability of $\mathrm{SAH}$.

Key Words: Hypertension. Body mass index. Waist Circumference. 


\section{INTRODUÇÃO}

A hipertensão arterial sistêmica (HAS) é caracterizada por níveis pressóricos elevados e sustentados. É uma doença crônica de alto impacto social devido à sua alta prevalência e baixas taxas de controle (VI DIRETRIZES BRASILEIRAS DE HIPERTENSÃO, 2010; NOBRE; SAMMOUR; SOBRINHO, 2011).

No Brasil, a principal causa de morte para homens e mulheres são as doenças cardiovasculares (DCV), sendo a HAS o mais importante fator de risco independente para o desenvolvimento de acidente vascular cerebral, doença arterial coronariana e insuficiência renal (MANSUR; FAVARATO, 2012; BARBARO et al., 2011; FUCHS; SILVA, 2011; ROSENDORFF et al., 2015). A HAS se expressa como um dos maiores fatores de redução de expectativa e qualidade de vida (PASSOS; ASSIS; BARRETO, 2006). Estima-se que $75 \%$ das mortes mundiais em 2030 serão causadas por doenças não transmissíveis e que 23,4 milhões serão devidas a DCV (WORLD HEALTH ORGANIZATION, 2008).

A obesidade é ocasionada por um desbalanço entre o ganho de peso e o gasto energético e há uma nítida associação entre o ganho ponderal e a maior prevalência de DCV (WORLD HEALTH ORGANIZATION, 1998; PEIXOTO et al., 2006). O excesso de peso e a obesidade central estão relacionados à HAS desde idades jovens (SBC, 2010; ROSENDORFF et al., 2015; NOBRE; SAMMOUR; SOBRINHO, 2011; BOMBELLI et al., 2011; WORLD HEALTH ORGANIZATION, 2008; GIERACH et al., 2014; HALPERN et al., 2002; OLIVEIRA et al., 2013; DOMINGOS et al., 2013).

O Índice de Massa Corpórea (IMC) pode ser utilizado para estimar a prevalência de obesidade em uma população. No entanto, por não considerar a variação de distribuição da gordura corporal, o IMC pode não corresponder ao mesmo risco para a saúde de cada indivíduo. Sabe-se que a adiposidade abdominal pode variar dramaticamente dentro de um estreito intervalo de IMC. Sendo assim, outro indicador valioso que reflete risco aumentado de doenças relacionadas com a obesidade é a Circunferência da Cintura (CC) (WORLD HEALTH ORGANIZATION, 2008; SCARPELLINI; CARVALHO; SANTOS-HISS, 2011; SILVA; PETROSKI; PERES, 2012; GIERACH 
et al., 2014). Além dos métodos de avaliação antropométrica descritos anteriormente, outras ferramentas como bioimpedância, ressonância magnética e a absorciometria por raios $X$ de dupla energia (DEXA) são métodos mais precisos na pesquisa de obesidade, mas o custo $\mathrm{e}$ as dificuldades práticas de tais técnicas limitam sua utilidade (SILVA; PETROSKI; PERES, 2012; PEIXOTO et al., 2005; ASSOCIAÇÃO BRASILEIRA PARA O ESTUDO DA OBESIDADE E DA SÍNDROME METABÓLICA, 2009).

A rotina de trabalho e fatores peculiares à profissão tornam os motoristas de caminhão uma população com grande vulnerabilidade às doenças metabólicas $\mathrm{e}$ cardiovasculares. O estado nutricional,

\section{METODOLOGIA}

Trata-se de um estudo transversal, desenvolvido a partir dos dados obtidos durante o período de março a junho de 2013 na campanha Saúde na Estrada, promovida pela Liga Acadêmica de Fisiologia do curso de medicina do Centro Universitário UnirG (LAF-UnirG). A amostra foi composta por 548 indivíduos, todos do sexo masculino. A pesquisa foi realizada em o sedentarismo e distúrbios do sono são determinantes destacáveis para o risco aumentado de doenças (CAVAGIONI et al., 2009). Existe associação entre sonolência e os principais componentes da síndrome metabólica, além de HAS e CC de modo independente (MANSUR et al., 2015). Baixa escolaridade e pouca orientação sobre hábitos saudáveis como atividade física e a alimentação, são fatores que dificultam o entendimento e adesão dos motoristas à promoção de saúde (ROCHA et al., 2015).

Considerando que o estilo de vida dos motoristas de caminhão favorece o ganho de peso, o objetivo do estudo foi avaliar associação entre IMC e CC e a ocorrência de HAS em caminhoneiros no norte do país.

um posto de combustível, localizado na BR-153, quilômetro 667, no município de Gurupi-TO. O estudo foi aprovado pelo Comitê de Ética em Pesquisa com Seres Humanos do Centro Universitário UnirG (parecer oㅜ 486.667).

A população estudada foi composta por motoristas que pernoitaram no local, convidados a participar da pesquisa de forma 
aleatória. Foram incluídos aqueles motoristas que estavam em pleno exercício da função laboral e que consentiram sua participação na pesquisa através da assinatura do Termo de Consentimento Livre e Esclarecido (TCLE). A coleta de dados foi obtida mediante a aplicação de questionário para caracterização sociodemográfica, realizada aferições das medidas antropométricas pretendidas e aferição da pressão arterial. Ao final de cada atendimento foram fornecidas, individualmente, orientações de saúde.

A aferição da pressão arterial foi realizada com esfigmomanômetro aneróide da marca $\mathrm{BD} \AA$, com precisão de $\pm 3 \mathrm{mmHg}$, devidamente calibrado. Os procedimentos para obtenção da pressão arterial foram aqueles recomendados para o método indireto na VI Diretrizes Brasileiras de Hipertensão. Após certificar o correto posicionamento do paciente e do manguito, foi utilizado o método palpatório do pulso radial para estimar a pressão sistólica e posterior aferição com o método auscultatório da artéria braquial. A pressão sistólica foi determinada pela ausculta do primeiro som (fase I de Korotkoff) e a pressão diastólica no desaparecimento dos sons (fase $\mathrm{V}$ de Korotkoff). Foram executadas duas medidas com intervalo mínimo de um minuto entre elas. Para fins de análise, foram utilizados os valores da segunda medida, sendo descartados os valores da primeira aferição. Foram considerados como hipertensos os indivíduos que apresentaram valores pressóricos $\geq 140 \mathrm{mmHg}$ para pressão arterial sistólica (PAS) e/ou $\geq 90 \mathrm{mmHg}$ para pressão arterial diastólica (PAD) (SBC, 2010).

A massa corporal foi determinada em balança antropométrica mecânica com capacidade de $150 \mathrm{~kg}$ e divisão de $100 \mathrm{~g}$, devidamente calibrada, com o avaliado de pé no centro da plataforma da balança, descalços e com a menor quantidade de objetos e acessórios possível, com os pés paralelos, os braços estendidos ao longo do corpo, a cabeça ereta, olhando para frente. A altura foi mensurada com o avaliado estando em pé, ereto, pés paralelos, calcanhares unidos, cintura pélvica, cintura escapular e occipital em contato com a haste do estadiômetro fixado à balança antropométrica e os braços estendidos ao longo do corpo, sem dobrar os joelhos. A medida foi realizada na inspiração máxima (BRASIL, 2004). 
Com as medidas de peso e altura, calculou-se o IMC, obtido pelo peso $(\mathrm{Kg})$ dividindo pela altura $\left(\mathrm{m}^{2}\right)$, tendo os seguintes pontos de corte: < $18,5 \mathrm{~kg} / \mathrm{m}^{2}$ (baixo peso); 18,5-24,9 $\mathrm{kg} / \mathrm{m}^{2}$ (normal); 25,0 a 29,9 kg/m² (Sobrepeso); $\geq 30 \mathrm{~kg} / \mathrm{m}^{2}$ (obesidade), para maiores de 20 anos e menor que 60 anos (WORLD HEALTH ORGANIZATION, 2000).

A CC foi aferida com fita métrica inelástica e flexível, com precisão de $0,1 \mathrm{~cm}$. A técnica utilizada para essa medida foi: o avaliado estando de pé, ereto, abdômen relaxado, braços estendidos ao longo do corpo e os pés separados. A roupa foi afastada, a fita passada ao redor da cintura no ponto médio entre o rebordo costal inferior e a crista ilíaca e a leitura foi realizada

\section{RESULTADOS}

A amostra foi composta por 548 indivíduos, todos do sexo masculino, predominantes na profissão de caminhoneiro. A média de idade foi de 44 anos $( \pm 10,5)$ e $59,5 \%$ estavam entre 30 e 49 anos. A maioria $(91,6 \%)$ dos entrevistados era das raças branca e parda $(45,8 \%$ cada). Em relação ao estado civil, $75,9 \%$ responderam ser casados ou estar em uma união imediatamente após uma expiração completa (BRASIL, 2004; SBC, 2005).

Os valores obtidos foram classificados conforme o risco de complicações metabólicas. Para homens: $\geq 94 \mathrm{~cm}$ - risco aumentado e $\geq 102 \mathrm{~cm}$ - risco aumentado substancialmente (SBC, 2005). A análise estatística dos dados foi descritiva, utilizando frequência absoluta e relativa. Para testar associação entre as variáveis foram utilizados os testes do Qui-quadrado, Qui-quadrado de tendência e exato de Fisher. Foi calculada razão de chances (oddsratio) com respectivo intervalo de confiança de $95 \%$ utilizando regressão logística. Foi considerado significativo $p<0,05$. A análise foi realizada no Stata 12.

estável. Quanto à renda mensal, 66,8\% disseram ter rendimento maior que 2.550 reais. Mais da metade dos pesquisados tiveram escolaridade correspondente ao ensino fundamental ou menos ( $\leq 8$ anos de estudo), sendo que $14,4 \%$ estudaram menos de 4 anos e $44,2 \%$ estudaram de 5 a 8 anos. 
Tabela 1. Prevalência de hipertensão arterial sistêmica e Razão de chance de hipertensão arterial sistêmica em motoristas de transporte de carga da BR-153, conforme variáveis sociodemográficas e antropométricas. Gurupi, Tocantins, Brasil, 2013.

\begin{tabular}{|c|c|c|c|c|}
\hline Variáveis & $\mathrm{N}$ total $(\%)$ & HAS N (\%) & OR (IC95\%) & Valor $\mathrm{p}^{\star}$ \\
\hline Idade & & & & 0,002 \\
\hline $20-29$ & $47(8,6)$ & $11(4,6)$ & 1 & \\
\hline $30-39$ & $168(30,7)$ & $73(30,5)$ & $2,51(1,20-5,28)$ & \\
\hline $40-49$ & $158(28,8)$ & $63(26,4)$ & $2,17(1,03-4,58)$ & \\
\hline$\geq 50$ & $175(31,9)$ & $92(38,5)$ & $3,63(1,74-7,58)$ & \\
\hline Raça & & & & 0,341 \\
\hline Branca & $251(45,8)$ & $112(46,9)$ & $0,74(0,39-1,39)$ & \\
\hline Parda & $251(45,8)$ & $103(43,1)$ & 0,64 & \\
\hline Preta & $46(8,39)$ & $24(10,0)$ & 1 & \\
\hline Estado civil & & & & 0,198 \\
\hline Solteiro & $96(17,5)$ & $34(14,2)$ & $1,50(0,95-2,38)$ & \\
\hline Casado/união estável & $416(75,9)$ & $188(78,7)$ & $1,63(0,75-3,55)$ & \\
\hline Separado/divorciado/viúvo & $36(6,57)$ & $17(7,1)$ & 1 & \\
\hline Renda mensal & & & & $0,799^{* *}$ \\
\hline$<R \$ 1.020,00$ & $19(3,5)$ & $7(2,93)$ & 1 & \\
\hline $\mathrm{R} \$ 1.020,00$ a $2.550,00$ & $163(29,7)$ & $73(30,5)$ & $1,39(0,52-3,71)$ & \\
\hline$>R \$ 2.550,00$ & $366(66,8)$ & $159(66,5)$ & $1,32(0,51-3,42)$ & \\
\hline Escolaridade & & & & 0,654 \\
\hline$<4$ anos & $79(14,4)$ & $36(15,1)$ & $1,44(0,74-2,79)$ & \\
\hline De 5 a 8 anos & $242(44,2)$ & $109(45,6)$ & $1,41(0,81-2,45)$ & \\
\hline De 9 a 11 anos & $159(29,0)$ & $69(28,9)$ & $1,32(0,74-2,37)$ & \\
\hline$>11$ anos & $68(12,41)$ & $25(10,5)$ & 1 & \\
\hline $\begin{array}{l}\text { Índice de Massa Corporal } \\
\left(\mathrm{kg} / \mathrm{m}^{2}\right)\end{array}$ & & & & $<0,001^{\star * *}$ \\
\hline$<25$ & $100(18,2)$ & $17(7,1)$ & 1 & \\
\hline 25-29,9 & $188(34,3)$ & $71(29,7)$ & $2,96(1,63-5,39)$ & \\
\hline $30-34,9$ & $183(33,4)$ & $102(42,7)$ & $6,15(3,38-11,18)$ & \\
\hline$\geq 35$ & $77(14,0)$ & $49(20,5)$ & $8,54(4,25-17,18)$ & \\
\hline Circunferência da cintura $(\mathrm{cm})$ & & & & $<0,001^{* * *}$ \\
\hline$<94$ & $148(27,0)$ & $28(11,7)$ & 1 & \\
\hline $94-101,9$ & $138(25,2)$ & $61(25,5)$ & $3,40(2,00-5,78)$ & \\
\hline$\geq 102$ & $262(47,8)$ & $150(62,8)$ & $5,74(3,56-9,26)$ & \\
\hline \multicolumn{5}{|l|}{${ }^{*}$ Qui-quadrado } \\
\hline \multicolumn{5}{|l|}{${ }^{* *}$ Teste exato de Fisher } \\
\hline \multicolumn{5}{|l|}{ *** Qui-quadrado de tendência } \\
\hline $\begin{array}{l}\text { OR - oddsratio, } \\
\text { IC } 95 \% \text { - intervalo de confiang }\end{array}$ & I & & & \\
\hline
\end{tabular}

A tabela 1 apresenta a caminhoneiros estavam com prevalência de HAS conforme as sobrepeso ou algum grau de obesidade variáveis sociodemográficas e $\quad(I M C \geq 25)$ e $73 \%$ estavam com a antropométricas dos caminhoneiros. circunferência da cintura aumentada, Quanto ao excesso de peso, $81,7 \%$ dos 
sendo $25,2 \%$ com CC $\geq 94 \mathrm{~cm}$ e $47,8 \%$ $\geq 102 \mathrm{~cm}$.

Foi detectado HAS em 239 indivíduos, correspondendo a 43,6\% da amostra. A porcentagem de indivíduos com HAS aumentou de acordo com aumento da idade, conforme mostrado na tabela 1.

A regressão logística (OR IC95\%) mostrou associação significativa $(p<0,001)$ entre o excesso de peso e HAS tanto para o IMC quanto para CC. A análise apontou aumento da probabilidade de HAS em 196\% para indivíduos com IMC entre 25 e 29 $\mathrm{Kg} / \mathrm{m}^{2}$, em $515 \%$ para IMC 30 e 34,9 $\mathrm{Kg} / \mathrm{m}^{2}$ e em $754 \%$ para aqueles com

\section{DISCUSSÃO}

O presente estudo mostrou a associação do excesso de peso e da obesidade, avaliados pelo IMC e CC, com a HAS em caminhoneiros. O excesso de peso, detectado pelos indicadores antropométricos, mostrouse em geral associado à elevação da pressão arterial.

A profissão de motorista de caminhão carateriza-se por longas horas de trabalho e horários irregulares, incluindo a noite de trabalho. Portanto, comumente esses
IMC $\geq 35 \mathrm{Kg} / \mathrm{m}^{2}$. Para a variável circunferência da cintura, a chance de HAS aumentou em $240 \%$ quando a CC foi $>94 \mathrm{~cm}$ e em $474 \%$ quando a CC foi $>102 \mathrm{~cm}$.

Apesar de os dois métodos antropométricos mostrarem associação significativa com HAS, com as categorias de IMC $\geq 30 \mathrm{Kg} / \mathrm{m}^{2}$ a probabilidade de o indivíduo apresentar HAS foi maior do que aquelas observadas para os níveis da CC. Contudo, nota-se que os dois métodos mostraram que quanto maior o excesso de peso, maior a chance de o indivíduo apresentar HAS.

profissionais têm limitações no que tange a um estilo de vida saudável. $\mathrm{O}$ ritmo de trabalho contribui para a privação crônica de sono e para obesidade, além expor o caminhoneiro a um acúmulo de outros fatores de risco modificáveis para DCV. A alta prevalência de obesidade nessa população, maus hábitos alimentares e sedentarismo, conferem chance aumentada para DCV, destancando-se a HAS, doenças metabólicas e gastrointestinais (MANSUR et al., 2015; 
ROCHA et al., 2015; MARQUEZE; ULHÔA; MORENO, 2012; HIRATA et al., 2011; PAULA; SILVA; GUEDES, 2008; HOFFMAN, 2003).

Nesse estudo, 239 indivíduos apresentaram HAS correspondendo a $43,6 \%$ da amostra estudada. Em outro estudo, 258 motoristas de transporte de cargas foram avaliados no trecho Paulista-Régis Bittencourt da Rodovia BR-116, constatando uma prevalência de HAS de 37\% (CAVAGIONI, et al., 2010). Já a prevalência de HAS no Brasil, mostrada em revisão de literatura, ficou entre $20 \%$ e $44 \%$, estando apenas $30 \%$ dos hipertensos com a pressão arterial controlada (PONTES-NETO et al., 2008). Passos, Assis e Barreto (2006) levantaram uma estimativa de prevalência de HAS a partir de 13 estudos populacionais publicados entre 1990 e 2004, evidenciando que cerca de $20 \%$ dos adultos brasileiros tinham hipertensão e com evidente tendência de aumento com a idade.

Um excedente no peso corporal total, oriundo do aumento de um ou mais dos seus constituintes (músculo, gordura, osso e água), cujos valores de IMC variam entre 26 e $29.9 \mathrm{Kg} / \mathrm{m}^{2}$, caracteriza 0 sobrepeso.

Diferentemente, a obesidade resulta de um aumento generalizado ou localizado da gordura em relação ao peso corporal, associado a um IMC maior ou igual a $30 \mathrm{Kg} / \mathrm{m}^{2}$. Nesse estudo, 81,7\% dos caminhoneiros apresentaram sobrepeso ou algum grau de obesidade (IMC $\geq 25 \mathrm{~kg} / \mathrm{m} 2$ ), o que sugere um risco preocupante quando comparado a dados do Instituto Nacional de Alimentação e Nutrição, que revelam aproximadamente $32 \%$ da população adulta brasileira com algum grau de sobrepeso (DOMINGOS et al., 2013; GUEDES; GUEDES, 2003).

O IMC e a CC são indicadores antropométricos amplamente utilizados na identificação de fatores de risco cardiovasculares (BECK; LOPES; PITANGA, 2011). Em relação ao valor preditivo desses indicadores para a ocorrência da HAS na amostra do presente estudo, tanto o IMC quanto a CC apontaram que a chance do indivíduo apresentar HAS se eleva significativamente à medida que a categoria do indicador aumenta. De acordo a tabela 1, as prevalências de obesidade total (IMC $\geq 30 \mathrm{~kg} / \mathrm{m} 2$ ) e obesidade abdominal $(\mathrm{CC} \geq 94 \mathrm{~cm}$ ) foram, respectivamente, de $63,2 \%$ e 88,3\%. Evidenciou-se que indivíduos com IMC $\geq 35 \mathrm{Kg} / \mathrm{m}^{2}$ têm 8,54 de chance a mais de ter HAS que aqueles com $\mathrm{IMC}<25 \mathrm{~kg} / \mathrm{m} 2$ e indivíduos com $C C \geq 102 \mathrm{~cm}$ têm 5,74 de chance a 
mais de ter HAS que aqueles com CC < $94 \mathrm{~cm}$. Estudos populacionais sugerem que $75 \%$ dos diagnósticos de hipertensão podem ser devidos diretamente à obesidade. Sabe-se ainda que a PA diminui com a perda de peso e se eleva tanto com seu ganho, quanto com o avançar da idade, como foi constatado neste estudo, em consonância com a literatura (BARBARO et al., 2011).

No município de São Paulo, a prevalência de HAS foi de 29,9\% em funcionários do sexo masculino de um hospital geral privado. Registrou-se também, aumento significativo da prevalência do agravo com o aumento da idade e para os que trabalhavam no período noturno. Nesse mesmo estudo, a HAS atribuível a CC e ao IMC foram $48 \%$ e $56 \%$, respectivamente (SARNO; MONTEIRO, 2007). Um estudo feito em Goiânia (GO), em 2001, mostrou que entre os obesos a proporção de homens hipertensos foi de 58,4\%, enquanto em indivíduos de IMC normal a proporção de hipertensos foi de 38,4\% (PEIXOTO et al., 2006).

As DCV caracterizam-se por possuir etiologia multifatorial. Entretanto, estudos epidemiológicos demonstram relações consistentes entre idade avançada e HAS, possivelmente justificadas pelo enrijecimento gradual da aorta, natural do envelhecimento (BARCELÓ; SAEZ; TUERO, 2009; MOULATLET et al., 2010; KAPLAN; OPIE, 2006; PEIXOTO et al., 2006). Essa relação mostra-se direta e linear, de maneira que para a faixa etária acima de 65 anos a prevalência de HAS é superior a $60 \%$, e chega a $75 \%$ em indivíduos com 70 anos ou mais (SBC, 2010; MOULATLET et al., 2010). Dentre os caminhoneiros estudados, aqueles com idade igual ou superior a 50 anos apresentaram o incremento de $263 \%$ de chance de HAS quando comparados aos indivíduos entre 20 e 29 anos.

$O$ excesso e a irregularidade das horas de trabalho, bem como o sedentarismo e maus hábitos alimentares, explicam a alta prevalência de obesidade entre os motoristas de caminhão. Em diversas populações, estudos transversais e prospectivos mostraram que o excesso de adiposidade corporal é um dos mais importantes fatores de risco para HAS, independentemente da idade (PEIXOTO, et al., 2006; HIRATA, et al., 2011).

Em se tratando de um estudo transversal, uma possível limitação seria a ausência de discriminação entre os indivíduos previamente diagnosticados com HAS e aqueles que 
apresentaram apenas um pico hipertensivo no momento da aferição, contrariando a VI Diretrizes Brasileiras de Hipertensão, cujo diagnóstico é validado por medidas repetidas da PA, em condições ideais, em pelo menos três ocasiões. Além disso, não foram discriminados aqueles hipertensos que,

\section{CONCLUSÃO}

Os resultados demonstram que os motoristas de transporte de carga que trafegam pela Rodovia BR-153 apresentam excesso de peso, obesidade total e central associados à HAS. Esses indivíduos estão diariamente expostos a fatores de risco cardiovasculares e, consequentemente, têm maior chance de morbidade e mortalidade, uma vez que a HAS está na origem de importantes DCV.

Os hábitos e estilos de vida são passíveis de intervenção, por isso medidas do setor público em saúde são necessárias de implementação na prática. Sugerimos que ações no momento da aferição, estavam com níveis pressóricos adequadamente controlados por medicações antihipertensivas. No entanto, essas considerações não anulam a constatação do uso do IMC e CC para rastreio de HAS.

preventivas educativas sejam realizadas para esse público, na busca de contribuir para a melhoria da saúde desses profissionais.

\section{AGRADECIMENTOS}

Agradecemos a Liga Acadêmica de Fisiologia do Centro Universitário UnirG (LAF-UnirG) e a direção do Auto Posto Décio em Gurupi-TO, por viabilizar a realização desse trabalho. Prestamos agradecimento especial à DSc. Nelita Gonçalves Faria de Bessa, professora de Pesquisa em Saúde, pela ajuda e incentivo

\section{REFERÊNCIAS}

Associação Brasileira para o Estudo da Obesidade e da Síndrome Metabólica. Diretrizes brasileiras de obesidade 2009/2010 / ABESO - Associação Brasileira para - Estudo da Obesidade e da Síndrome Metabólica. - 3.ed. - Itapevi, SP : AC Farmacêutica, 2009. 
BARBARO, Natália Ruggeriet al. Fisiopatologia da hipertensão no diabetes e na obesidade. Revista Brasileira de Hipertensão,Campinas, v. 18, n. 3, p.89-94, 2011.

BARCELÓ, Maria Antònia; SAEZ, Marc; TUERO, Gabriel Coll. Individual socioeconomic factors conditioning cardiovascular disease risk. American Journal of Hypertension, New York, v. 22, n. 10, p. 1085 - 1095, 2009.

BECK, Carmem Cristina; LOPES, Adair da Silva; PITANGA, Francisco José Gondim. Indicadores Antropométricos como Preditores de Pressão Arterial Elevada em Adolescentes. Arquivos Brasileiros de Cardiologia, Florianópolis, v. 12, n. 96, p.126-133, jun. 2011.

BOMBELLI, M. et al. Impact of Body Mass Index and Waist Circumference on the Long-Term Risk of Diabetes Mellitus, Hypertension, and Cardiac Organ Damage. Hypertension, v. 58, n. 6, p.1029-1035, 24 out. 2011.

CAVAGIONI, Luciane Cesira et al . Agravos à saúde, hipertensão arterial e predisposição ao estresse em motoristas de caminhão. Revista da Escola de Enfermagem de USP, São Paulo, v. 43, n. spe2, p. 1267-1271, Dec. 2009.

DOMINGOS, Everton et al. Associação entre estado nutricional antropométrico, circunferência de cintura e pressão arterial em adolescentes. Revista Brasileira de Cardiologia, Londrina, v. 02, n. 26, p.94-99, abr. 2013.

DOMINGOS, Josélia Benedita Carneiro. Consumo de álcool, sobrepeso e obesidade entre caminhoneiros. Revista Enfermagem UERJ, Rio de Janeiro, v. 18, n. 3, p. 377382, jul/set. 2010.

FUCHS, Sandra C.; SILVA, Andressa Alves da. Hipertensão arterial e diabetes mellitus: uma visão global. Revista Brasileira de Hipertensão, Porto Alegre, v. 18, n. 13, p.83-89, set. 2011.

GIERACH, Marcin et al. Correlation between Body Mass Index and Waist Circumference in Patients with Metabolic Syndrome. ISRN Endocrinology, v. 2014, p.1-6, 2014. Hindawi Publishing Corporation.

GUEDES, Dartagnan Pinto; GUEDES, Joana Elisabete Ribeiro Pinto. Controle do peso corporal: composição corporal, atividade física e nutrição. $2 a$ ed. Rio de Janeiro: Shape; 2003.

HALPERN, Alfredo et al . Diretrizes para Cardiologistas sobre Excesso de Peso e Doença Cardiovascular dos Departamentos de Aterosclerose, Cardiologia Clínica e FUNCOR da Sociedade Brasileira de Cardiologia.Arquivos. Brasileiros Cardiologia, São Paulo , v. 78, supl. 1, p. 01-13, 2002.

HIRATA, Raquel Pastrélloet al., Prevalência de obesidade e hipertensão arterial em uma população de motoristas profissionais rodoviários interestaduais de ônibus. Con. Scientiae Saúde, v. 10, n. 3, p. 494-499, 2011. 
HOFFMAN, André Luiz. Qualidade de vida dos motoristas de caminhão usuários do Programa Rodopac: um estudo de caso [dissertação de mestrado]. Florianópolis(SC): Universidade Federal de Santa Catarina; 2003.

KAPLAN, Norman M.; OPIE, Lionel H. Controversies in cardiology 2: controversies in hypertension. Lancet, London, v. 367, n. 9505, p. 168-176, 2006.

MANSUR, Antonio de Paduaet al. Risk Factors for Cardiovascular Disease, Metabolic Syndrome and Sleepiness in Truck Drivers. Arquivos Brasileiros de Cardiologia, São Paulo, v. 6, n. 105, p.560-565, 2015.

MANSUR, Antonio de Padua; FAVARATO, Desidério. Mortalidade por Doenças Cardiovasculares no Brasil e na Região Metropolitana de São Paulo: Atualização 2011. Arquivos Brasileiros de Cardiologia, São Paulo, v. 22, n. 2, p.755-761, 2012.

MARQUEZE, Elaine C; A ULHÔA, Melissa; MORENO, Claudia R C. Irregular working times and metabolic disorders among truck drivers: a review. Work, São Paulo, v. 41, n. 1, p.3718-3725, 2012.

MUNARETTI, Deonilde BalduÍno et al. Hipertensão arterial referida e indicadores antropométricos de gordura em idosos. Revista da Associação Médica Brasileira, Florianópolis, v. 57, n. 1, p.25-30, out. 2011

NOBRE, Luciana Neri; SAMMOUR, Simone Nascimento Fagundes; COSTA SOBRINHO, Paulo de Souza. Índice de massa corporal e circunferência de cintura como preditores de pressão arterial alterada em adolescentes. Revista Médica de Minas Gerais, Barbacena, v. 21, n. 4, p.404-412, 2011.

OLIVEIRA, Luciano Machado Ferreira Tenório de et al . Associação entre obesidade geral e abdominal com a hipertensão arterial em idosas ativas. Revista de Educação Física UEM, Maringá, v. 24, n. 4, p. 659-668, Dec. 2013.

PASSOS, Valéria Maria de Azeredo; ASSIS, Tiago Duarte; BARRETO, Sandhi Maria. Hipertensão arterial no Brasil: estimativa de prevalência a partir de estudos de base populacional. Epidemiologia e Serviços de Saúde, Brasília, v. 15, n. 1, p.35-45, mar. 2006.

PAULA, A. C.; SILVA, A. M. C.; GUEDES, H. M. O uso dos serviços de atenção básica a saúde por caminhoneiros. 2008. Monografia (Graduação de Enfermagem) - Faculdade de Enfermagem, Centro Universitário do Leste de Minas Gerais, Ipatinga, 2008.

PEIXOTO, Maria do Rosário Gondim et al . Circunferência da cintura e índice de massa corporal como preditores da hipertensão arterial. Arquivos Brasileiros de Cardiologia, São Paulo, v. 87, n. 4, p. 462-470, Oct. 2006

PONTES-NETO, Octávio Marques et al. Stroke Awareness in Brazil: Alarming Results in a Community-Based Study. Stroke, v. 39, n. 2, p.292-296, 2007. 
ROCHA, Elias Marcelino da.Prevalência de obesidade e sedentarismo em caminhoneiros. 2015. Revista Univar, v.1, n.13, pag 165-169, 2015.

ROSENDORFF, Clive MD et al. Treatment of hypertension in patients with coronary artery disease: a scientific statement from the American Heart Association, American College of Cardiology, and American Society of Hypertension. Journal of the American College of Cardiology, v. 65, p.1998-2038, 2015.

SARNO, Flávio; MONTEIRO, Carlos Augusto. Importância relativa do índice de massa corporal e da circunferência abdominal na predição da hipertensão arterial. Revista de Saúde Pública, São Paulo, v.41, n.5, p.788-96, 2007.

SCARPELLINI, Ennio da Silveira; CARVALHO, Eduardo Elias Vieira de; SANTOSHISS, Michele Daniela Borges dos. Associação entre circunferência abdominal e hipertensão arterial em mulheres com segmento nas equipes de saúde da família no município de bebedouro/sp. Revista Epeq Fafibe, v. 1, p. 81-85, 2011.

SILVA, Diego Augusto Santos; PETROSKI, Edio Luiz; PERES, Marco Aurelio. Is high body fat estimated by body mass index and waist circumference a predictor of hypertension in adults? A population-based study. Nutrition Journal, v. 11, n. 1, 2012.

SOCIEDADE BRASILEIRA DE CARDIOLOGIA (SBC). I Diretriz Brasileira de Diagnóstico e Tratamento da Síndrome Metabólica. Arquivos Brasileiros de Cardiologia. MCMXLIII: São Paulo, Volume 84, Suplemento I, Abril 2005. 27p.

SOCIEDADE BRASILEIRA DE CARDIOLOGIA (SBC). VI Diretrizes Brasileiras de Hipertensão. Arquivos Brasileiros de Cardiologia. São Paulo, v. 95, n. 1, supl. 1, p. I-III, 2010 .

WORLD HEALTH ORGANIZATION. Waist circumference and waist-hip ratio: report of a WHO expert consultation, Geneva, 8-11 December 2008. 2011. World Health Organization.

WORLD HEALTH ORGANIZATION. Obesity: preventing and managing the global epidemic.Report of a WHO Consultation on Obesity. Geneva, 1998. 